\title{
Pacific
}

Journal of

Mathematics

\section{ON OVERTWISTED, RIGHT-VEERING OPEN BOOKS}

\author{
PAOLO Lisca
}




\title{
ON OVERTWISTED, RIGHT-VEERING OPEN BOOKS
}

\author{
PAOLO LisCA
}

\begin{abstract}
We exhibit infinitely many overtwisted, right-veering, non-destabilizable open books, thus providing infinitely many counterexamples to a conjecture of Honda, Kazez and Matić. The page of all our open books is a four-holed sphere and the underlying 3-manifolds are lens spaces.
\end{abstract}

\section{Introduction}

The purpose of this note is to construct infinitely many counterexamples to a conjecture of Honda, Kazez and Matić from [Honda et al. 2009]. For the basic notions of contact topology not recalled below we refer the reader to [Etnyre 2003; Geiges 2008].

Let $S$ be a compact, oriented surface with boundary and $\operatorname{Map}(S, \partial S)$ the group of orientation-preserving diffeomorphisms of $S$ that restrict to $\partial S$ as the identity, up to isotopies fixing $\partial S$ pointwise. An open book (also known as an abstract open book) is a pair $(S, \Phi)$ where $S$ is a surface as above and $\Phi \in \operatorname{Map}(S, \partial S)$. Giroux [2002] introduced a fundamental operation of stabilization $(S, \Phi) \rightarrow\left(S^{\prime}, \Phi^{\prime}\right)$ on open books, and proved the existence of a 1-1 correspondence between the set of open books modulo stabilization and the set of contact 3-manifolds modulo isomorphism (see, for example, [Etnyre 2006] for details). Honda, Kazez and Matić [Honda et al. 2007] showed that a contact 3-manifold is tight if and only if it corresponds to an equivalence class of open books $(S, \Phi)$ all of whose monodromies $\Phi$ are right-veering (in the sense of [Honda et al. 2007, Section 2]). In [Goodman 2005; Honda et al. 2007] it is also showed that every open book can be made right-veering after a sequence of stabilizations. Honda, Kazez and Matić [Honda et al. 2009] proved that when $S$ is a holed torus, the contact structure corresponding to $(S, \Phi)$ is tight if and only if $\Phi$ is right-veering, and conjectured that a non-destabilizable right-veering open book corresponds to a tight contact 3-manifold. The HondaKazez-Matić conjecture was recently disproved by Lekili [2011], who produced a counterexample $(S, \Phi)$ with $S$ equal to a four-holed sphere and whose underlying 3-manifold is the Poincaré homology sphere.

MSC2010: primary 57R17; secondary 53D10.

Keywords: contact surgery, destabilizable diffeomorphisms, Giroux's correspondence, open books, overtwisted contact structures, right-veering diffeomorphisms. 
We shall now describe our examples. Denote by $\delta_{\gamma} \in \operatorname{Map}(S, \partial S)$ the class of a positive Dehn twist along a simple closed curve $\gamma \subset S$.

Theorem 1.1. Let $S$ be an oriented four-holed sphere, and $a, b, c, d$, e the simple closed curves on $S$ shown in Figure 1.

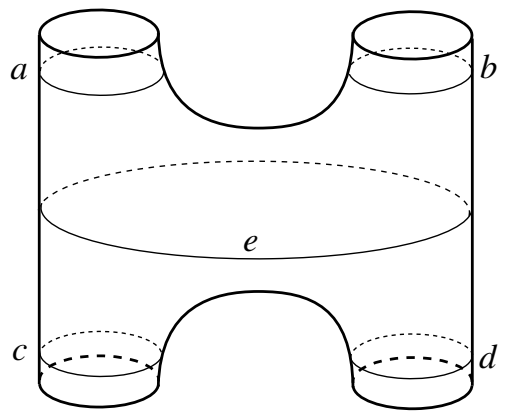

Figure 1. The four-holed sphere $S$.

Let $h, k \geq 1$ be integers. Define $\Phi_{h, k}:=\delta_{a}^{h} \delta_{b} \delta_{c} \delta_{d} \delta_{e}^{-k-1} \in \operatorname{Map}(S, \partial S)$. Then

- the underlying 3-manifold $Y_{\left(S, \Phi_{h, k}\right)}$ is the lens space

$$
L((h+1)(2 k-1)+2,(h+1) k+1) ;
$$

- the associated contact structure $\xi_{\left(S, \Phi_{h, k}\right)}$ is overtwisted;

- $\Phi_{h, k}$ is right-veering;

- $\left(S, \Phi_{h, k}\right)$ is not destabilizable.

Warning: in the above statement we adopt the convention that the lens space $L(p, q)$ is the oriented 3-manifold obtained by performing a rational surgery along an unknot in $S^{3}$ with coefficient $-p / q$.

We prove Theorem 1.1 in Section 2. The proof can be outlined as follows. In Proposition 2.1 we use elementary arguments to determine a contact surgery presentation for the contact 3-manifold $\left(Y_{\left(S, \Phi_{h, k}\right)}, \xi_{\left(S, \Phi_{h, k}\right)}\right)$, and in Corollary 2.2 we apply Proposition 2.1 and a few Kirby calculus moves to identify the underlying 3manifold $Y_{\left(S, \Phi_{h, k}\right)}$. In Proposition 2.3 we appeal to calculations from [Lekili 2011] to deduce that the contact Ozsváth-Szabó invariant of $\xi_{\left(S, \Phi_{h, k}\right)}$ vanishes, and we conclude from the fact that $Y_{\left(S, \Phi_{h, k}\right)}$ is a lens space that $\xi_{\left(S, \Phi_{h, k}\right)}$ must be overtwisted. That $\Phi_{h, k}$ is right-veering in Lemma 2.4 follows directly from [Arıkan and Durusoy 2012, Theorem 4.3], but it can also be deduced by imitating the proof of [Lekili 2011, Theorem 1.2], that is, by applying [Honda et al. 2007, Corollary 3.4]. Finally, we use results from [Arıkan 2008; Lekili 2011] to conclude that $\left(S, \Phi_{h, k}\right)$ is not destabilizable. 


\section{Proof of Theorem 1.1}

Recall that every contact structure has a contact surgery presentation. We refer the reader to [Ding and Geiges 2004] for this fact and the basic properties of contact surgeries, and to [Lisca and Stipsicz 2004] for the use of the "front notation" in contact surgery presentations, in particular for the meaning of Figure 2 below.

Proposition 2.1. For $h, k \geq 1$, the contact structure $\xi_{\left(S, \Phi_{h, k}\right)}$ has the contact surgery presentation given by Figure 2.

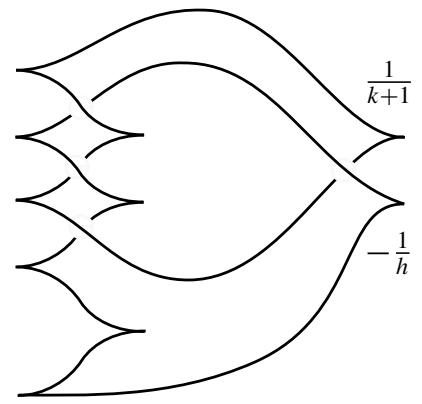

Figure 2. Contact surgery presentation for $\xi_{\left(S, \Phi_{h, k}\right)}, h, k \geq 1$.

Proof. Figure 3 (a) represents an open book $(A, f)$, where $A$ is an annulus and $f$ is a positive Dehn twist along the core of $A$. The associated contact 3-manifold is the standard contact 3 -sphere $\left(S^{3}, \xi_{\text {st }}\right)$, the annulus $A$ can be viewed as the page of an open book decomposition of $S^{3}$, and the curve $\kappa$ in the picture can be made Legendrian via an isotopy of the contact structure, in such a way that the contact framing on $\kappa$ coincides with the framing induced on it by the page (see [Etnyre 2006, Figure 11]). The knot $\kappa$ is the unique Legendrian unknot in $\left(S^{3}, \xi_{\mathrm{st}}\right)$ having Thurston-Bennequin invariant $\operatorname{tb}(\kappa)=-1$ and rotation number $\operatorname{rot}(\kappa)=0$. A suitable choice of orientation for $\kappa$ uniquely specifies its negative oriented Legendrian stabilization $\kappa_{-}$, which satisfies $\operatorname{tb}\left(\kappa_{-}\right)=-2$ and $\operatorname{rot}\left(\kappa_{-}\right)=-1$. As shown in [Etnyre 2006], $\kappa_{-}$can be realized as sitting on the page of a Giroux stabilization $\left(A^{\prime}, f^{\prime}\right)$ of $(A, f)$. This is illustrated in Figure $3(\mathrm{~b})$, assuming the orientation on $\kappa$ was taken to be "counterclockwise" in Figure 3 (a). Finally, Figure 3 (c) shows an open book $\left(S, f^{\prime \prime}\right)$ obtained by Giroux stabilizing $\left(A^{\prime}, f^{\prime}\right)$ and containing both $\kappa_{-}$and $\left(\kappa_{-}\right)_{-}$in $S\left(\kappa_{-}\right.$was also given the "counterclockwise" orientation in Figure $3(\mathrm{~b}))$. Clearly $\left(S, f^{\prime \prime}\right)$ still corresponds to $\left(S^{3}, \xi_{\mathrm{st}}\right)$, and it is well-known that $\kappa_{-},\left(\kappa_{-}\right)_{-}$are the two Legendrian knots illustrated in Figure 2 (when oriented "clockwise" in that picture). By definition, $\Phi_{h, k}$ is obtained by precomposing $f^{\prime \prime}$ with $k+1$ negative Dehn twists along parallel copies of $\kappa_{-}$and $h$ positive Dehn twists along parallel copies of $\left(\kappa_{-}\right)_{-}$. Moreover, if $m \neq 0$ is an integer, $\frac{1}{m}$-contact 
surgery along any Legendrian knot $\lambda$ is equivalent to $\frac{m}{|m|}$-contact surgeries along $|m|$ Legendrian push-offs of $\lambda$ [Ding and Geiges 2004]. Since page and contact framings coincide, and by [Etnyre 2006, Theorem 5.7] positive (negative, respectively) Dehn twists correspond to -1 -contact surgeries (+1-contact surgeries, respectively), it is easy to check that the resulting contact structure is given by the contact surgery presentation of Figure 2.

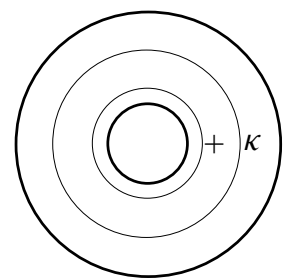

(a)

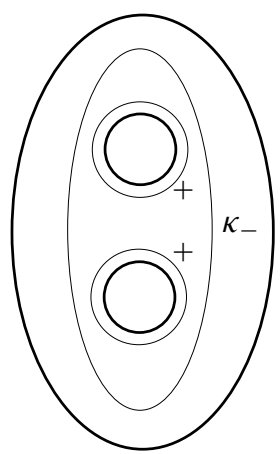

(b)

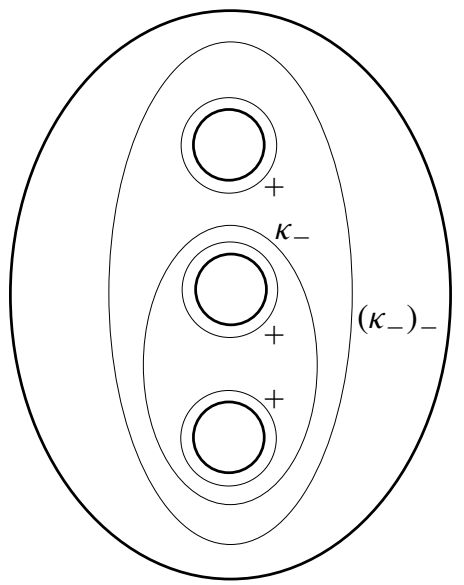

(c)

Figure 3. Determination of the contact surgery presentation.

Corollary 2.2. For $h, k \geq 1$, the oriented 3-manifold underlying the open book $\left(S, \Phi_{h, k}\right)$ is the lens space $L((h+1)(2 k-1)+2,(h+1) k+1)$.

Proof. Using the fact that the two Legendrian unknots illustrated in Figure 2 have Thurston-Bennequin invariants -2 and -3 , it is easy to check that the topological surgery underlying Figure 2 is given by the first (upper left) picture of Figure 4. Two +1-blowups and two inverse slam-dunks give the second picture, while the

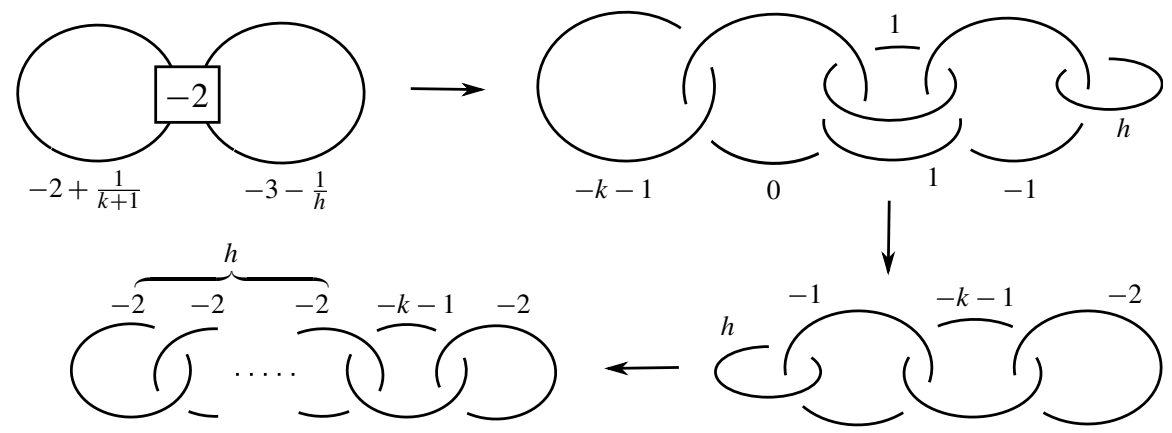

Figure 4. Determination of the underlying 3-manifold. 
third picture is obtained from the second one by sliding the -1-framed knot over the 0 -framed knot and then applying two +1 -blow-downs. The last picture is obtained simply converting the $h$-framed unknot in the third picture into the string of -2framed unknots via a sequence of -1 -blowups and a final +1 -blowdown. The last picture shows that the underlying 3-manifold $Y_{\left(S, \Phi_{h, k}\right)}$ is obtained by performing a rational surgery on an unknot in $S^{3}$ with coefficient $-p / q$, where

$$
\frac{p}{q}=2-\frac{1}{k+1-\frac{1}{2-\frac{1}{\ddots \cdot \frac{1}{2}}}}=\frac{(h+1)(2 k-1)+2}{(h+1) k+1} .
$$

Therefore, according to our conventions $Y_{\left(S, \Phi_{h, k}\right)}$ can be identified with the lens space $L((h+1)(2 k-1)+2,(h+1) k+1)$.

Proposition 2.3. For $h, k \geq 1$, the contact structure $\xi_{\left(S, \Phi_{h, k}\right)}$ is overtwisted.

Proof. By [Giroux 2000; Honda 2000] a contact structure on a lens space is either overtwisted or Stein fillable. Moreover, Stein fillable contact structures have nonzero contact Ozsváth-Szabó invariant [Ozsváth and Szabó 2005]. Finally, [Lekili 2011, Theorem 1.3] immediately implies that the contact invariant of $\left(S, \Phi_{h, k}\right)$ vanishes, therefore $\xi_{\left(S, \Phi_{h, k}\right)}$ must be overtwisted.

Lemma 2.4. For $h, k \geq 1$, the diffeomorphism class

$$
\Phi_{h, k}=\delta_{a}^{h} \delta_{b} \delta_{c} \delta_{d} \delta_{e}^{-k-1} \in \operatorname{Map}(S, \partial S)
$$

is right-veering.

Proof. The lemma follows immediately from the statement of Theorem 4.3 in [Arıkan and Durusoy 2012]. Alternatively, one can imitate the proof of Theorem 1.2 of [Lekili 2011]. Indeed, applying Corollary 3.4 from [Honda et al. 2007] to the monodromy $\Phi_{1}=\delta_{e}^{-k-1}$ and a properly embedded arc $\gamma_{c d} \subset S$ disjoint from the curve $e$ and connecting the components $\partial_{c}$ and $\partial_{d}$ of $\partial S$ parallel to the curves $c$ and $d$ shows that $\Phi_{2}=\delta_{d} \delta_{e}^{-k-1}$ is right-veering with respect to $\partial_{d}$. Another application of the corollary to $\Phi_{2}$ and $\gamma_{c d}$ shows that $\Phi_{3}=\delta_{c} \delta_{d} \delta_{e}^{-k-1}$ is rightveering with respect to $\partial_{c}$. Moreover, since $\delta_{c}$ is right-veering with respect to $\partial_{c}$ and the composition of right-veering diffeomorphisms is still right-veering [Honda et al. 2007], $\Phi_{3}$ is right-veering with respect to $\partial_{d}$ as well. Appying the corollary in the same way to $\Phi_{3}$ and an arc connecting the components of $\partial S$ parallel to the curves $a$ and $b$ yields the statement of the lemma. 
Proof of Theorem 1.1. Corollary 2.2, Proposition 2.3 and Lemma 2.4 establish the first three portions of the statement. Thus we only need to show that $\left(S, \Phi_{h, k}\right)$ is not destabilizable for every $h, k \geq 1$. If $\left(S, \Phi_{h, k}\right)$ were destabilizable, it would be a stabilization of an open book $\left(S^{\prime}, \Phi^{\prime}\right)$, where $S^{\prime}$ is a three-holed sphere and $\Phi^{\prime}=\tau_{1}^{a_{1}} \tau_{2}^{a_{2}} \tau_{3}^{a_{3}}$, where $a_{i} \in \mathbb{Z}$ and $\tau_{i}$ is a positive Dehn twist along a simple closed curve parallel to the $i$-th boundary components of $S^{\prime}, i=1,2,3$. By [Arrkan 2008, Theorem 1.2], $\xi_{\left(S, \Phi_{h, k}\right)}$ is tight if and only if $a_{i} \geq 0, i=1,2,3$. Therefore, by Proposition 2.3 at least one of these exponents must be strictly negative. But the proof of Theorem 1.2 of [Lekili 2011] shows that when one of the $a_{i}$ 's is negative, any stabilization of $\left(S^{\prime}, \Phi^{\prime}\right)$ to an open book with page a four-holed sphere is not right-veering. This would contradict Lemma 2.4, therefore we conclude that $\left(S, \Phi_{h, k}\right)$ cannot be destabilizable.

Note added in proof: after the submission of the present paper the author was informed of unpublished work of A. Wand containing, in particular, a different proof of Proposition 2.3.

\section{Acknowledgements}

I wish to thank Yanki Lekili for pointing out to me his paper [Lekili 2011]. The present work is part of the author's activities within CAST, a Research Network Program of the European Science Foundation.

\section{References}

[Arıkan 2008] M. F. Arıkan, "Planar contact structures with binding number three", pp. 90-124 in Proceedings of Gökova Geometry-Topology Conference 2007, edited by S. Akbulut et al., GGT, Gökova, 2008. MR 2010h:57038 Zbl 1193.57013

[Arıkan and Durusoy 2012] M. F. Arıkan and S. Durusoy, "On the classification of certain planar contact structures", Acta Math. Hungar. 134:4 (2012), 529-542. Zbl 06028279

[Ding and Geiges 2004] F. Ding and H. Geiges, "A Legendrian surgery presentation of contact 3-manifolds", Math. Proc. Cambridge Philos. Soc. 136:3 (2004), 583-598. MR 2005m:57038 Zbl 1069.57015

[Etnyre 2003] J. B. Etnyre, "Introductory lectures on contact geometry", pp. 81-107 in Topology and geometry of manifolds (Athens, GA, 2001), edited by G. Matić and C. McCrory, Proc. Sympos. Pure Math. 71, Amer. Math. Soc., Providence, RI, 2003. MR 2005b:53139 Zbl 1045.57012

[Etnyre 2006] J. B. Etnyre, "Lectures on open book decompositions and contact structures", pp. 103-141 in Floer homology, gauge theory, and low-dimensional topology, edited by D. A. Ellwood et al., Clay Math. Proc. 5, Amer. Math. Soc., Providence, RI, 2006. MR 2007g:57042 Zbl 1108.53050

[Geiges 2008] H. Geiges, An introduction to contact topology, Cambridge Studies in Advanced Mathematics 109, Cambridge University Press, 2008. MR 2008m:57064 Zbl 1153.53002

[Giroux 2000] E. Giroux, "Structures de contact en dimension trois et bifurcations des feuilletages de surfaces”, Invent. Math. 141:3 (2000), 615-689. MR 2001i:53147 Zbl 1186.53097 
[Giroux 2002] E. Giroux, "Géométrie de contact: de la dimension trois vers les dimensions supérieures", pp. 405-414 in Proceedings of the International Congress of Mathematicians, Vol. II (Beijing, 2002), edited by T. Li, Higher Ed. Press, Beijing, 2002. MR 2004c:53144 Zbl 1015.53049

[Goodman 2005] N. Goodman, "Overtwisted open books from sobering arcs", Algebr. Geom. Topol. 5 (2005), 1173-1195. MR 2006h:57022 Zbl 1090.57020

[Honda 2000] K. Honda, "On the classification of tight contact structures. I", Geom. Topol. 4 (2000), 309-368. MR 2001i:53148 Zbl 0980.57010

[Honda et al. 2007] K. Honda, W. H. Kazez, and G. Matić, "Right-veering diffeomorphisms of compact surfaces with boundary", Invent. Mathem. 169:2 (2007), 427-449. MR 2008e:57028 Zbl 1167.57008

[Honda et al. 2009] K. Honda, W. H. Kazez, and G. Matić, "On the contact class in Heegaard Floer homology", J. Differential Geom. 83:2 (2009), 289-311. MR 2011f:57050 Zbl 1186.53098

[Lekili 2011] Y. Lekili, "Planar open books with four binding components", Algebr. Geom. Topol. 11:2 (2011), 909-928. MR 2012f:57059 Zbl 1220.57017

[Lisca and Stipsicz 2004] P. Lisca and A. I. Stipsicz, "Ozsváth-Szabó invariants and tight contact three-manifolds. I", Geom. Topol. 8 (2004), 925-945. MR 2005e:57069 Zbl 1059.57017

[Ozsváth and Szabó 2005] P. Ozsváth and Z. Szabó, "Heegaard Floer homology and contact structures", Duke Math. J. 129:1 (2005), 39-61. MR 2006b:57043 Zbl 1083.57042

Received July 26, 2011.

PAOLO LISCA

Dipartimento di Matematica "L. TONelli”

UNIVERSITÀ DI PISA

LARgo BRUno PONTECORVO, 5

56127 PISA

ITALY

lisca@dm.unipi.it 


\title{
PACIFIC JOURNAL OF MATHEMATICS
}

\author{
http://pacificmath.org \\ Founded in 1951 by \\ E. F. Beckenbach (1906-1982) and F. Wolf (1904-1989)
}

\section{EDITORS}

V. S. Varadarajan (Managing Editor)

Department of Mathematics

University of California

Los Angeles, CA 90095-1555

pacific@math.ucla.edu

Vyjayanthi Chari

Department of Mathematics

University of California

Riverside, CA 92521-0135

chari@math.ucr.edu

\section{Robert Finn}

Department of Mathematics Stanford University

Stanford, CA 94305-2125

finn@math.stanford.edu

Kefeng Liu

Department of Mathematics

University of California

Los Angeles, CA 90095-1555

liu@math.ucla.edu
Darren Long

Department of Mathematics

University of California

Santa Barbara, CA 93106-3080

long@math.ucsb.edu

Jiang-Hua Lu

Department of Mathematics

The University of Hong Kong

Pokfulam Rd., Hong Kong jhlu@maths.hku.hk

Alexander Merkurjev

Department of Mathematics

University of California

Los Angeles, CA 90095-1555

merkurev@math.ucla.edu
Sorin Popa

Department of Mathematics University of California

Los Angeles, CA 90095-1555 popa@math.ucla.edu

Jie Qing

Department of Mathematics

University of California

Santa Cruz, CA 95064

qing@cats.ucsc.edu

Jonathan Rogawski

Department of Mathematics

University of California

Los Angeles, CA 90095-1555

jonr@math.ucla.edu

\section{PRODUCTION}

pacific@math.berkeley.edu

\section{SUPPORTING INSTITUTIONS}

ACADEMIA SINICA, TAIPEI

CALIFORNIA INST. OF TECHNOLOGY INST. DE MATEMÁTICA PURA E APLICADA KEIO UNIVERSITY

MATH. SCIENCES RESEARCH INSTITUTE NEW MEXICO STATE UNIV.

OREGON STATE UNIV.

\author{
STANFORD UNIVERSITY \\ UNIV. OF BRITISH COLUMBIA \\ UNIV. OF CALIFORNIA, BERKELEY \\ UNIV. OF CALIFORNIA, DAVIS \\ UNIV. OF CALIFORNIA, LOS ANGELES \\ UNIV. OF CALIFORNIA, RIVERSIDE \\ UNIV. OF CALIFORNIA, SAN DIEGO \\ UNIV. OF CALIF., SANTA BARBARA
}

\author{
UNIV. OF CALIF., SANTA CRUZ \\ UNIV. OF MONTANA \\ UNIV. OF OREGON \\ UNIV. OF SOUTHERN CALIFORNIA \\ UNIV. OF UTAH \\ UNIV. OF WASHINGTON \\ WASHINGTON STATE UNIVERSITY
}

These supporting institutions contribute to the cost of publication of this Journal, but they are not owners or publishers and have no responsibility for its contents or policies.

See inside back cover or pacificmath.org for submission instructions.

The subscription price for 2012 is US \$420/year for the electronic version, and \$485/year for print and electronic.

Subscriptions, requests for back issues from the last three years and changes of subscribers address should be sent to Pacific Journal of Mathematics, P.O. Box 4163, Berkeley, CA 94704-0163, U.S.A. Prior back issues are obtainable from Periodicals Service Company, 11 Main Street, Germantown, NY 12526-5635. The Pacific Journal of Mathematics is indexed by Mathematical Reviews, Zentralblatt MATH, PASCAL CNRS Index, Referativnyi Zhurnal, Current Mathematical Publications and the Science Citation Index.

The Pacific Journal of Mathematics (ISSN 0030-8730) at the University of California, c/o Department of Mathematics, 969 Evans Hall, Berkeley, CA 94720-3840, is published monthly except July and August. Periodical rate postage paid at Berkeley, CA 94704, and additional mailing offices. POSTMASTER: send address changes to Pacific Journal of Mathematics, P.O. Box 4163, Berkeley, CA 94704-0163.

PJM peer review and production are managed by EditFLOW ${ }^{\mathrm{TM}}$ from Mathematical Sciences Publishers.

PUBLISHED BY PACIFIC JOURNAL OF MATHEMATICS

at the University of California, Berkeley 94720-3840

A NON-PROFIT CORPORATION

Typeset in LATEX

Copyright $(02012$ by Pacific Journal of Mathematics 


\section{PACIFIC JOURNAL OF MATHEMATICS}

Volume $257 \quad$ No. $1 \quad$ May 2012

Energy and volume of vector fields on spherical domains

FABiano G. B. BRito, ANDRÉ O. Gomes and Giovanni S. Nunes

Maps on 3-manifolds given by surgery

BOLDIZSÁR KALMÁR and ANDRÁS I. STIPSICZ

Strong solutions to the compressible liquid crystal system

Yu-Ming ChU, Xian-Gao LiU and XIAO LIU

Presentations for the higher-dimensional Thompson groups $n V$

Johanna HenNig and FranCESCo MatuCCI

Resonant solutions and turning points in an elliptic problem with oscillatory

boundary conditions

ALFONSO CASTRO and ROSA PARDO

Relative measure homology and continuous bounded cohomology of topological pairs

\section{Roberto Frigerio and CRISTINA PAgLiAnTINi}

Normal enveloping algebras

ALEXANDRE N. GrishKov, Marina RASSKazova and SALVATORE SICILIANO

Bounded and unbounded capillary surfaces in a cusp domain

YASUNORI AOKI and DAVID SIEGEL

On orthogonal polynomials with respect to certain discrete Sobolev inner product

Francisco Marcellán, Ramadan Zejnullahu, Bujar Fejzullahu and EDMUNDO HUERTAS

Green versus Lempert functions: A minimal example

PASCAL THOMAS

Differential Harnack inequalities for nonlinear heat equations with potentials under the Ricci flow

JIA-YONG WU

On overtwisted, right-veering open books

PAOLO LISCA

Weakly Krull domains and the composite numerical semigroup ring $D+E\left[\Gamma^{*}\right]$

JUNG WOOK LIM

Arithmeticity of complex hyperbolic triangle groups 